\title{
Mirror-Image Asymmetry in Monozygotic Twins with Kabuki Syndrome
}

\author{
A. Riess $^{a} \quad$ A. Dufke ${ }^{a}$ O. Riess ${ }^{a} \quad$ S. Beck-Woedl ${ }^{a} \quad$ B. Fode ${ }^{b} \quad$ H. Skladny ${ }^{c}$ \\ R. Klaes ${ }^{c}$ A. Tzschach ${ }^{a}$ \\ a Institute of Human Genetics and b University Children's Hospital, University of Tuebingen, Tuebingen, and \\ ${ }^{c}$ Centre for Human Genetics, Mannheim, Germany
}

\section{Key Words}

Deletion · Kabuki syndrome • Mirror-image asymmetry •

MLL2 $\cdot$ Monozygotic twins

\begin{abstract}
Kabuki syndrome (OMIM 147920) is a rare disorder characterised by moderate intellectual disability, growth retardation, microcephaly and characteristic facial dysmorphic features which comprise long palpebral fissures, eversion of the lateral third of the eyelids and arched eyebrows with lateral sparseness. Mutations in MLL2 are the most frequent cause of this disorder. More than 100 MLL2 point mutations have been reported, but large intragenic deletions comprising one or more exons have not yet been identified. We report on a pair of monozygotic twin brothers in whom a deletion of 2 neighbouring exons was detected. The twins had the characteristic facial features of Kabuki syndrome, and they suffered from microcephaly, cleft lip and palate and congenital heart disease. Cleft lip and palate were left-sided in the first twin and right-sided in the second twin, i.e. they represented a mirror-image asymmetry. The intragenic deletion in these brothers broadens the spectrum of MLL2 mutations, and they provide a rare example of mirror-image asymmetry of congenital malformations in monozygotic twins.
\end{abstract}

\section{KARGER}

Fax +4161306 1234

E-Mail karger@karger.ch

www.karger.com
(C) 2012 S. Karger AG, Basel

1661-8769/12/0032-0094\$38.00/0

Accessible online at:

www.karger.com/msy
Kabuki syndrome (OMIM 147920) is a rare disorder characterised by mild to moderate intellectual disability, growth retardation, microcephaly and characteristic facial dysmorphic features which comprise long palpebral fissures, eversion of the lateral third of the eyelids and arched eyebrows with lateral sparseness. Heterozygous loss-of-function mutations in MLL2 have been identified in up to $74 \%$ of patients with Kabuki syndrome [Ng et al., 2010; Hannibal et al., 2011; Li et al., 2011; Micale et al., 2011; Paulussen et al., 2011; Banka et al., 2012]. To date, more than 100 point mutations have been detected in $M L L 2$, but large deletions comprising several exons have not yet been reported. MLL2 (OMIM 602113) encodes a SET-domain containing histone methyltransferase which plays a role in epigenetic transcriptional regulation [FitzGerald and Diaz, 1999]. Recently, mutations in the X-chromosomal gene KDM6A were detected in 3 male patients, demonstrating the genetic heterogeneity of Kabuki syndrome [Lederer et al., 2012].

Mirror-image asymmetry, e.g. the opposing orientation of hair whorls, is present in up to $25 \%$ of monozygotic twins and is assumed to be the result of a relatively late cleavage of the zygote [Burn, 1991]. In rare cases, mirror-image asymmetry has also been reported for congenital malformations such as cardiac defects, microtia or 

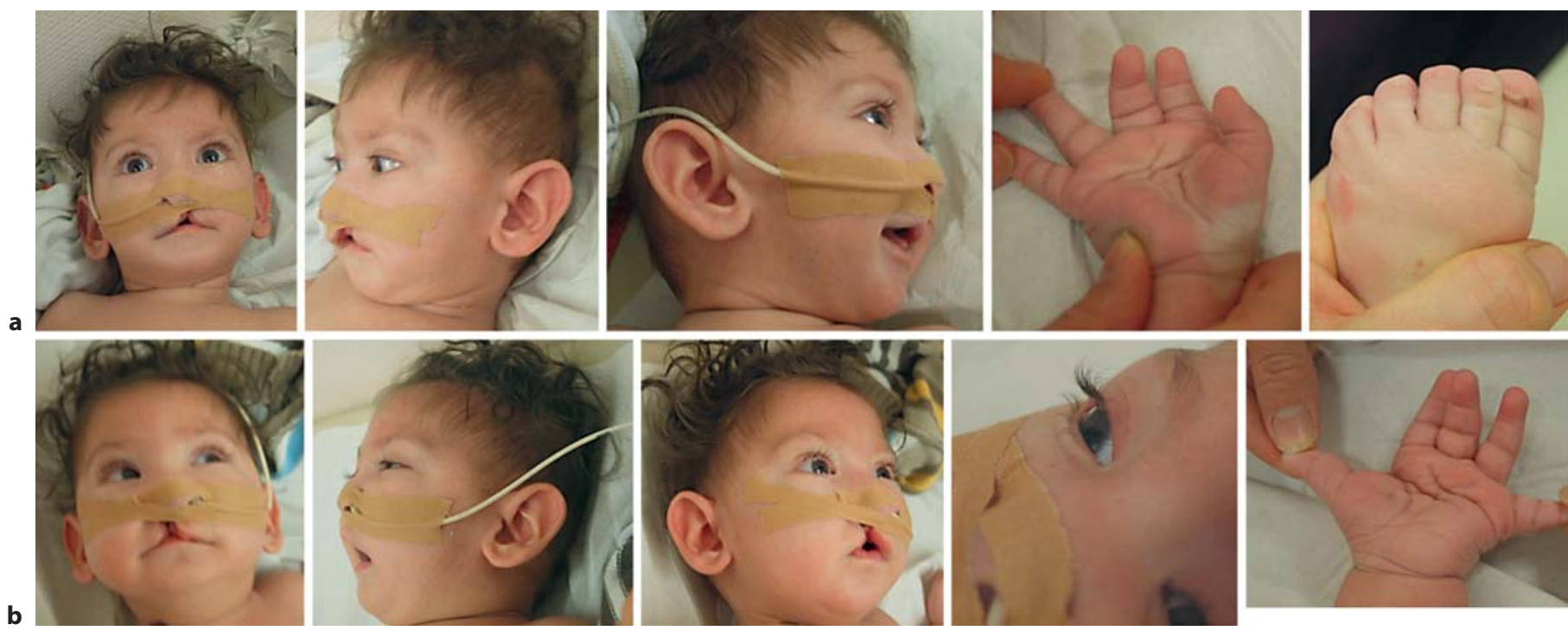

Fig. 1. Twin 1 (a) and twin 2 (b) at the age of 7 months. Note cleft lip, cup-shaped ears, long palpebral fissures, arched eyebrows, long eyelashes, eversion of lower eyelid, brachydactyly, deep palmar creases and finger pads.

dental anomalies [Sperber et al., 1994; Thacker et al., 2009; Brent, 2011].

Here, we report on a pair of identical twin brothers with Kabuki syndrome and mirror-image cleft lip and palate in whom we detected a deletion of 2 adjacent exons of MLL2.

\section{Clinical Report}

The twin boys were born by Caesarean section in the $32+6$ gestational week to healthy and non-consanguineous Turkish parents. They had 1 older healthy brother. Cardiac defects, cleft lip and microcephaly in both children had been detected during the pregnancy by ultrasound examination, and prenatal chromosome analyses and array CGH analysis revealed normal results.

Twin 1

Birth measurements of the first twin were normal: birth weight $1,660 \mathrm{~g}$ (15th centile), body length $43 \mathrm{~cm}$ (30th centile), occipitofrontal circumference (OFC) $28.5 \mathrm{~cm}$ (8th centile). He had a leftsided cleft lip and cleft palate. Congenital heart defect (small ventricular septal defect and atrial septal defect) was surgically corrected at the age of 7 months. Ultrasound examinations revealed duplicated kidneys of the left side. He suffered from congenital hypothyroidism, hypoglycemia, hyperbilirubinemia and feeding problems. On examination at the age of 10 months, he had persistent fingerpads, deep palmar creases, arched eyebrows, long eyelashes, and eversion of the lower eyelids (fig. 1a). He also had brachydactyly, hypoplastic nails and cup-shaped ears. Weight $(7,750 \mathrm{~g}$, 50 th centile) and length $(71 \mathrm{~cm}, 50$ th centile) were in the normal range, but he was microcephalic (OFC $42.5 \mathrm{~cm},<3$ rd centile).
Twin 2

Birth measurements of the second twin were normal: birth weight $1,945 \mathrm{~g}$ (40th centile), body length $42 \mathrm{~cm}$ (20th centile), OFC $29 \mathrm{~cm}$ (10th centile). He had a right-sided cleft lip and cleft palate and congenital heart defect (small ventricular septal defect and atrial septal defect). Ophthalmologic examination revealed a coloboma of the optic nerve of the right eye. He suffered from congenital hypothyroidism and cryptorchidism. Feeding problems necessitated the insertion of a percutaneous endoscopic gastrostomy tube. On examination at the age of 10 months, he had persistent fingerpads, deep palmar creases, long eyelashes, eversion of the lower eyelids, arched eyebrows, brachydactyly and hypoplastic nails (fig. 1b). He was microcephalic (OFC $42.5 \mathrm{~cm},<3 \mathrm{rd}$ centile); weight $(8,900 \mathrm{~g}$, 50th centile) and length $(70 \mathrm{~cm}, 50$ th centile) were within the normal range. At the age of 11 months he had epileptic seizures.

\section{Material and Methods}

DNA samples of the patients and their parents were isolated from peripheral blood leukocytes using routine procedures. Molecular genetic analysis of the 54 coding exons and flanking intronic regions of MLL2 was performed by Sanger sequencing. PCR conditions and primer sequences are available on request.

Multiplex ligation-dependent probe amplification (MLPA) was performed using the MRC-Holland Kit P389 (MRC-Holland, Amsterdam, The Netherlands) following the instructions of the manufacturer. This kit contains 27 probes which are evenly distributed over the length of MLL2. MLPA testing of the index patient (first twin) was performed 4 times, and testing of his brother and the parents were performed twice. 
Fig. 2. Results of the MLPA analysis of 27 $M L L 2$ probes. The 2 exons which are deleted in the twin brothers are framed in red.

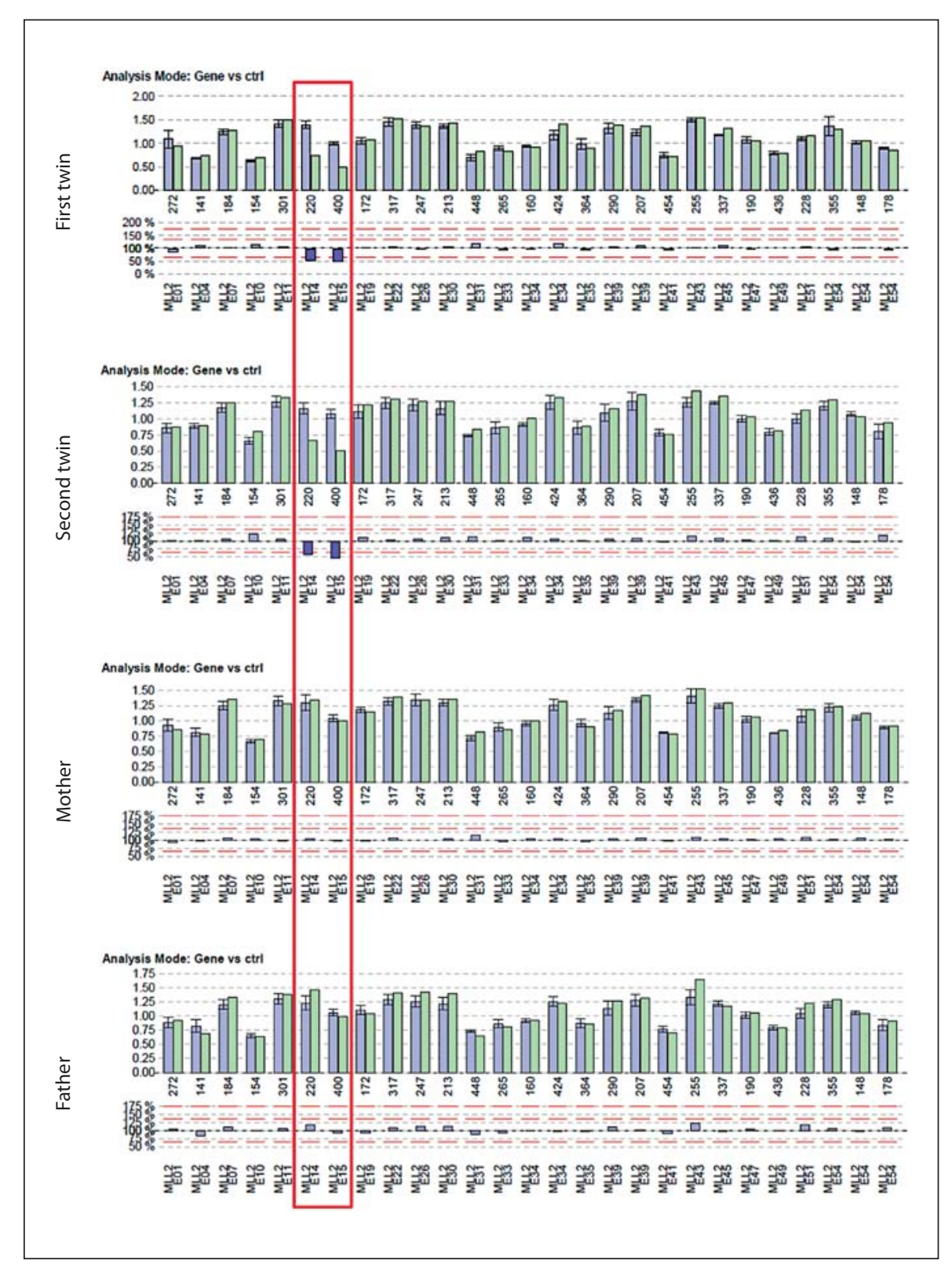

To determine whether the twins were monozygotic or dizygotic, 15 highly polymorphic autosomal short tandem repeat (STR) markers were tested using the PowerPlex ${ }^{\mathrm{TM}} 16$ system (Promega, Madison, Wisc., USA) according to the manufacturer's instructions.

\section{Results}

Sequencing of the MLL2 exons in the index patient (twin 1) failed to reveal any aberrations. MLPA analysis revealed a deletion of exons 14 and 15 of MLL2 (fig. 2).
The identical deletion was also present in the second twin, but not in the parents, i.e. it was a de novo deletion of 2 neighbouring exons. MLPA analysis of exons 11 and 19 revealed normal results. Since exons 12 and 13 and exons 16-18 were not tested, the deletion of exons 14 and 15 represents the minimal size of this deletion, and the maximal size could span exons 12 to 18 .

STR marker analysis demonstrated that the brothers were a pair of identical (monozygotic) twins, i.e. all markers had the same status in both twins. 


\section{Discussion}

The twin brothers reported here not only had the characteristic facial features of Kabuki syndrome, but they also suffered from congenital heart disease (CHD) and cleft lip and palate. In a recent survey of a large cohort of patients with Kabuki syndrome, cleft lip and palate and $\mathrm{CHD}$ had a higher prevalence in patients with truncating MLL2 mutations in comparison to those with missense mutations ( $43 \%$ vs. $21 \%$ for cleft lip and palate; and $55 \%$ vs. 30\% for CHD) [Hannibal et al., 2011]. Thus, the clinical features of the patients reported here are in accordance with the deletion of 2 or possibly more MLL2 exons which presumably leads to nonsense-mediated decay of the $M L L 2$ transcript. In contrast to other monogenic disorders such as Duchenne/Becker muscular dystrophy in which up to $70 \%$ of patients have intragenic DMD deletions [Takeshima et al., 2010], such deletions are apparently rare in $M L L 2$. Whereas more than $100 M L L 2$ point mutations are known, not a single $M L L 2$ deletion has been reported to date [ $\mathrm{Ng}$ et al., 2010; Hannibal et al., 2011; Li et al., 2011; Micale et al., 2011; Paulussen et al., 2011; Banka et al., 2012]. The dearth of larger deletions involving MLL2 might explain why array CGH studies failed to identify the causative gene for Kabuki syndrome, which was eventually achieved by applying whole-exome next-generation sequencing [ $\mathrm{Ng}$ et al., 2010]. In contrast, $K D M 6 A$, the second gene associated with Kabuki syn- drome, was identified by array CGH analyses, and all 3 KDM6A mutations that have been reported to date were partial or complete deletions of this gene [Lederer et al., 2012].

Mirror-image asymmetry of the cleft lip is a remarkable feature of this pair of identical twins (fig. 1). Mirrorimage asymmetry is present in up to $25 \%$ of monozygotic twins, and its usual manifestation is the opposing orientation of hair whorls and cowlicks [Newman, 1928; Burn, 1991]. Unilateral congenital malformations or dysmorphic features such as microtia, dental anomalies, eye disorders or cleft lip/palate constitute rare but more obvious examples of this phenomenon [Sperber et al., 1994; Satoh et al., 1995; Aknin et al., 2007; Brent, 2011]. Mirrorimage asymmetry is thought to be caused by a relatively late cleavage of the zygote, typically between day 9 and 12 after fertilization [Burn, 1991]. Cleavage at an even later point of time would result in conjoined twins [Burn, 1991].

In conclusion, the patients reported here broaden the spectrum of MLL2 mutations and constitute a rare example of mirror-image asymmetry of congenital malformations in monozygotic twins.

\section{Acknowledgement}

We thank the parents of the patients for their support.

\section{References}

Aknin C, Allart JF, Rouland JF: Unilateral keratoconus and mirror image in a pair of monozygotic twins [in French]. J Fr Ophtalmol 30 899-902 (2007).

-Banka S, Veeramachaneni R, Reardon W, Howard E, Bunstone S, et al: How genetically heterogeneous is Kabuki syndrome?: MLL2 testing in 116 patients, review and analyses of mutation and phenotypic spectrum. Eur J Hum Genet 20:381-388 (2012).

Brent B: Repair of microtia with sculpted rib cartilage grafts in identical, mirror-image twins: a case study. Ann Plast Surg 66:62-64 (2011).

-Burn J: Disturbance of morphological laterality in humans. Ciba Found Symp 162:282-299 (1991).

-FitzGerald KT, Diaz MO: MLL2: A new mammalian member of the trx/MLL family of genes. Genomics 59:187-192 (1999).

-Hannibal MC, Buckingham KJ, Ng SB, Ming JE, Beck AE, et al: Spectrum of MLL2 (ALR) mu- tations in 110 cases of Kabuki syndrome. Am J Med Genet A 155:1511-1516 (2011).

Lederer D, Grisart B, Digilio MC, Benoit V, Crespin M, et al: Deletion of KDM6A, a histone demethylase interacting with MLL2, in three patients with Kabuki syndrome. Am J Hum Genet 90:119-124 (2012).

Li Y, Bögershausen N, Alanay Y, Simsek Kiper PO, Plume N, et al: A mutation screen in patients with Kabuki syndrome. Hum Genet 130:715-724 (2011).

Micale L, Augello B, Fusco C, Selicorni A, Loviglio MN, et al: Mutation spectrum of $M L L 2$ in a cohort of Kabuki syndrome patients. Orphanet J Rare Dis 9:38 (2011).

Newman HH: Studies of human twins: II. Asymmetry reversal or mirror imaging in identical twins. Biol Bull 55:298-315 (1928).

Ng SB, Bigham AW, Buckingham KJ, Hannibal MC, McMillin MJ, et al: Exome sequencing identifies MLL2 mutations as a cause of Kabuki syndrome. Nat Genet 42:790-793 (2010).
Paulussen AD, Stegmann AP, Blok MJ, Tserpelis D, Posma-Velter C, et al: MLL2 mutation spectrum in 45 patients with Kabuki syndrome. Hum Mutat 32:E2018-2025 (2011).

Satoh K, Shibata Y, Tokushige H, Onizuka T: A mirror image of the first and second branchial arch syndrome associated with cleft lip and palate in monozygotic twins. Br J Plast Surg 48:601-605 (1995).

- Sperber GH, Machin GA, Bamforth FJ: Mirrorimage dental fusion and discordance in monozygotic twins. Am J Med Genet 51:4145 (1994).

Takeshima Y, Yagi M, Okizuka Y, Awano H, Zhang Z, et al: Mutation spectrum of the dystrophin gene in 442 Duchenne/Becker muscular dystrophy cases from one Japanese referral center. J Hum Genet 55:376-388 (2010).

Thacker D, Gruber PJ, Weinberg PM, Cohen MS: Heterotaxy syndrome with mirror image anomalies in identical twins. Congenit Heart Dis 4:50-53 (2009). 